\title{
MR UROGRAPHY WITH DYNAMIC 3D MIP AIDED DETECTION- DIAGNOSTIC SIGNIFICANCE VALUE IN SUSPECTED PELVICALIECTASIS CONFIRMATION
}

\author{
Ameen M. D1, Vetriraj $S^{2}$ \\ ${ }^{1}$ Associate Professor, Department of Radiodiagnosis. \\ ${ }^{2}$ Associate Professor, Department of Radiodiagnosis.
}

\begin{abstract}
BACKGROUND
\section{ABSTRACT}

Hydronephrosis is the most common cause of chronic renal failure. Hydronephrosis due to calculus disease is more frequent than without non-calculus aetiology. Mild hydronephrosis or mild pelvicaliectasis is commonly encountered on routine ultrasound screening. Radiologist's bias in mild pelvicaliectasis as to rule out physiological or early pathology is our purpose of study. Role of MR Urography serve as a problem solving tool for ultrasound in mild pelvicaliectasis by specific protocol with 3D aided and dynamic imaging.
\end{abstract}

The objective of this study is to evaluate efficacy of MR urography for suspecting hydronephrosis in asymptomatic and incidental detected hydronephrosis in other radiological modalities.

\section{MATERIALS AND METHODS}

34 patients were enrolled in descriptive study with institutional ethical review obtained. Clinically suspected pelvicaliectasis patients screened by Ultrasound were set as inclusion criteria. Pelvicaliectasis positive patients on ultrasound were subjected to MR urography and renal diuretic nuclear study. Remaining patients were set as control group and compared with the results. MR urography non-contrast protocol were deployed. The three dimensional sequence was used to track passage of contrast from kidneys to ureter and bladder. MR urography followed by furosemide scintigraphy was also done to compare the efficacy of MR urography, keeping as reference standard. Nuclear medicine furosemide renal study was done for all MR urography within 1 week duration. Renal functional test parameters like renal transit time and average contrast wash-out time are calculated, and in all the cases these parameters are depicted as characteristics of analysis curve. Bland-Altman plots were used to quantify intra- and interobserver performance. Statistical significance was derived by comparing both modality curves with a mean under the curve of 0.90. Percentage analysis was also done by comparing two modalities, sensitivity and specificity.

\section{RESULTS}

6 positive cases were noted from MR Urography from sample size $(\mathrm{N}=19)$ with left UPJ obstruction $(\mathrm{N}=1)$, right VUJ obstruction $(\mathrm{N}=1)$, stricture in right mid ureter $(\mathrm{n}=3)$ and left mid ureter $(\mathrm{N}=1)$. Calculus obstruction patients were excluded from the study. All the cases and results were compared with diuretic renal scintigraphy. On the basis of interobserver performance on characteristic curves, results obtained were almost similar. RTT obstructed (RTT $>410$ seconds) obtained from both characteristic curves with percentage analysis were with better sensitivity $(98.8 \%)$ and specificity $(100 \%)$.

\section{CONCLUSION}

MR Urography proved to be the future gold standard for suspected mild pelvicaliectasis in our study. However, large cohort study needs to be addressed to prove efficacy in large sample volume.

\section{KEYWORDS}

MR Urography; Pelvicaliectasis; Furosemide Scintigraphy; Ultrasound.

HOW TO CITE THIS ARTICLE: Ameen MD, Vetriraj S. MR urography with dynamic 3D MIP aided detection- diagnostic significance value in suspected pelvicaliectasis confirmation. J. Evolution Med. Dent. Sci. 2018;7(01):29-32, DOI: 10.14260/jemds/2018/8

\section{BACKGROUND}

Since its development in the late 1970s, diuretic renography has been a commonly used test for diagnosing suspected hydronephrosis. $(1,2,3)$ In some urological centres however excretory urography (IVP) is still used, as it is considered an equally reliable test.(4) In addition, isotope imaging facilities are not available everywhere. Although, in urologic practice diuretic isotope renal scan is recommended as the most useful procedure for diagnosis of mild pelvicaliectasis.

'Financial or Other Competing Interest': None.

Submission 05-12-2017, Peer Review 17-12-2017,

Acceptance 19-12-2017, Published 01-01-2018.

Corresponding Author:

Dr. Ameen M. $D$

Flat $1 C$, No. 9 ,

Swaraj Manor Leithe Castle Centre Street,

Santhome, Chennai-600028.

E-mail: drameenmdrd@rocketmail.com

DOI: $10.14260 /$ jemds $/ 2018 / 8$
Magnetic resonance urography (MRU) techniques have matured and have become applicable to the diagnosis of more and more diseases in urinary tract. Modern MRU offers not only increased spatial and temporal resolution, but also provides anatomic and functional information on renal perfusion, excretion and drainage. Patients with renal colic are better examined by non-enhanced computed tomography (CT) scanning, because it is highly sensitive in detecting stones. However, one disadvantage of non-enhanced CT is the high radiation dosage.

MRU is free of radiation and contrast media risks, so it is recommended to be a primary investigation in patients with no excretory function, in pregnant women, in children, in young persons and in those with contrast medium allergy.[5,2,3,6,7,8,4]

The most common MR urographic techniques for displaying the urinary tract can be divided into two categories: Static-fluid MR urography and Excretory MR urography. Static-fluid MR urography makes use of heavily 
T2-weighted sequences to image the urinary tract as a static collection of fluid, can be repeated sequentially (cine MR urography) to better demonstrate the ureters in their entirety and to confirm the presence of fixed stenosis, and is most successful in patients with dilated or obstructed collecting systems. Excretory MR urography is performed during the excretory phase of enhancement after the intravenous administration of gadolinium-based contrast material; thus, the patient must have sufficient renal function to allow the excretion and even distribution of the contrast material. Diuretic administration is an important adjunct to excretory MR urography, which can better demonstrate nondilated systems. Static-fluid and excretory MR urography can be combined with conventional MR imaging for comprehensive evaluation of the urinary tract. The successful interpretation of MR urographic examinations requires familiarity with the many pitfalls and artifacts that can be encountered with these techniques.

Hydronephrosis is the most common cause of chronic renal failure due to either calculus or non-calculus conditions. Hydronephrosis due to calculus disease is more frequent than without non-calculus aetiology. Mild hydronephrosis or mild pelvicaliectasis is commonly encountered on routine ultrasound screening. Radiologist's bias in mild pelvicaliectasis as to rule out physiological or early pathology is our purpose of study. Role of MR Urography is to serve as a problem solving tool for ultrasound in mild pelvicaliectasis by specific protocol with 3D aided and dynamic imaging. Future prospects with MR Urography, further studies on mild pelvicaliectasis problem solving condition is to be explored and our study also targets mild pelvicaliectasis condition.

\section{MATERIALS AND METHODS}

34 patients were enrolled in the descriptive study with institutional ethical review obtained. Clinically suspected pelvicaliectasis patients screened by Ultrasound were set as inclusion criteria. Pelvicaliectasis positive patients on ultrasound were subjected to MR urography and renal diuretic nuclear study. Remaining patients were set as control group and compared with the results.

\section{Statistical Analysis}

The collected data were analysed with IBM SPSS Statistics Software 23.0 Version. Bland-Altman plots were used to quantify intra- and inter-observer performance and statistical significance was derived by comparing both modality curves with a mean under the curve of 0.90 . Percentage analysis was also done by comparing two modalities, sensitivity and specificity.

\section{Image Protocol \\ MR Urography}

Satisfactory MR urograms were obtained with $1.5 \mathrm{~T}$ scanner, Siemens with 16 channel body coil.

Static-fluid MR urograms technique was deployed, obtained with single-shot fast spin-echo techniques in $1-2$ seconds.

Multiple images were obtained sequentially in a relatively short period of time and played as a cine loop.

T2-weighted imaging was performed by the following technique. For fat-suppressed T2-weighted imaging of the renal parenchyma and pelvic organs, we preferred a respiratory-triggered fast spin-echo sequence. For standard non-fat suppressed T1-weighted imaging, in-phase and opposed-phase gradient-echo sequences were used. For cine imaging of the ureters a thick-slab, heavily T2-weighted single-shot fast spin-echo sequence similar to sequences used for MR cholangiopancreatography is performed. This sequence is typically performed $10-15$ times with $5-10$ seconds between acquisitions to prevent tissue saturation. The total number of thick slab acquisitions can be varied to fit the circumstance.

No contrast imaging technique has been used in MR Urography study.

Renal nuclear study- Lasix response curves were obtained by using an intravenous injection of $99 \mathrm{~m} \mathrm{Tc}-\mathrm{MAG}_{3}$. Lasix was administered in each case $(\mathrm{N}=19)$ at 18 minutes and the two response curves analysis were obtained for two kidneys with ureter status and bladder.

\section{Image Analysis and Interpretation}

Two radiologists, independent of each other without bias reported MR Urography and Furosemide nuclear study. Characteristic analysis by RTT time and mean wash-out time of contrast into bladder were calculated by two modalities in seconds/ point of difference calculated.

In our study also, the right kidney was more commonly involved than the left, male: female ratio was $3: 1$.

\section{RESULTS}

Out of 19 patients, 13 patients were normal in both MR Urography and Furosemide nuclear study. Out of 6 positive cases noted from MR Urography from sample size $(\mathrm{N}=19)$ were left UPJ obstruction. Figure 4, showing pathology numbers involved in our cases.
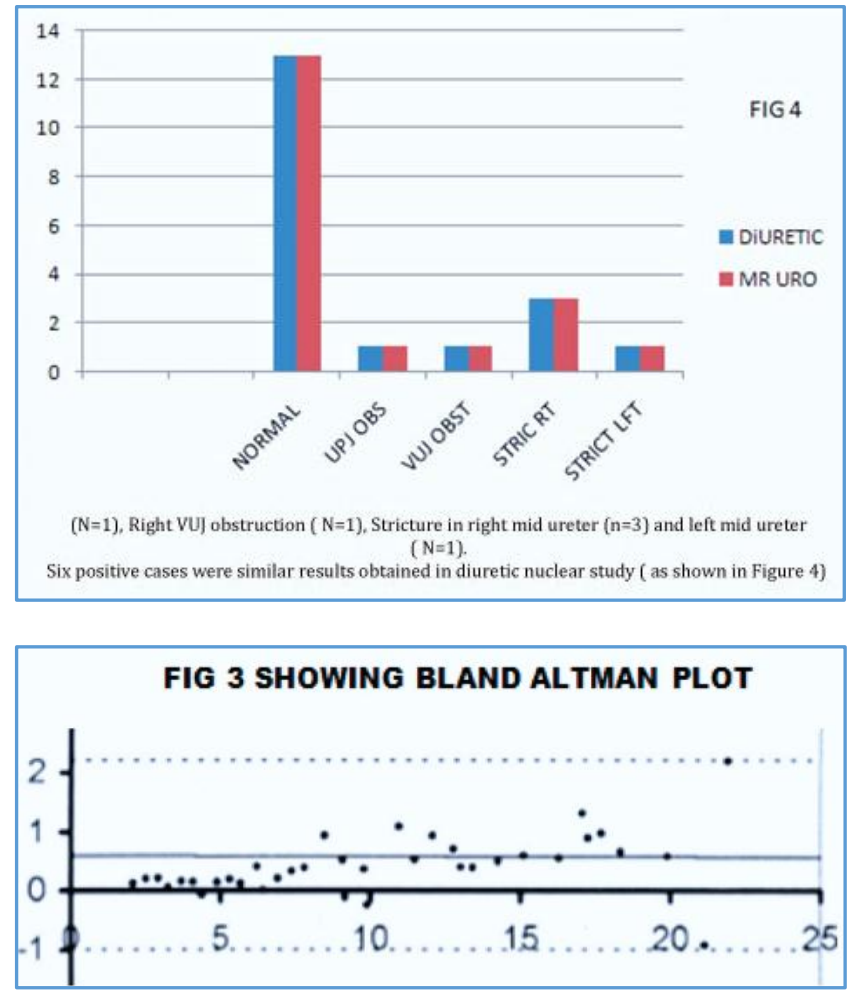

Figure 3, showing Bland-Altman plot mean \pm standard deviation derived among interobserver variation as results 
were similar. Only difference calculated from renal transit time (RTT) and average contrast wash-out time in bladder.

On the basis of interobserver performance on characteristic curves, results obtained were almost similar.

\begin{tabular}{|c|c|c|}
\hline Table 1 & Study 1 & Study 2 \\
\hline & Diuretic Study & MR Uro \\
\hline NORMAL & 13 & 13 \\
\hline UPJ OBS & 1 & 1 \\
\hline VUJ OBST & 1 & 1 \\
\hline STRICT RT & 3 & 3 \\
\hline STRICT LFT & 1 & 1 \\
\hline
\end{tabular}

Table 1, above showing similar results from both the modalities with minor variation. RTT obstructed (RTT $>410$ seconds) obtained from both characteristic curves with percentage analysis were better with sensitivity (98.8\%) and specificity (100\%).

\section{DISCUSSION}

As with any MR imaging technique, one must be aware of potential pitfalls when interpreting findings at MR urography.(6) When reviewing MR urographic images created with MIP or VR algorithms, one should always consult the original data (source images) to ensure that small filling defects are not obscured by surrounding high signal intensity urine.

MRU is able to make the distinction of physiological renal dilation from obstruction due to calculi causes. MRU is therefore a safer and sensitive alternative to conventional imaging techniques in detecting urinary stones in selected groups of patients.

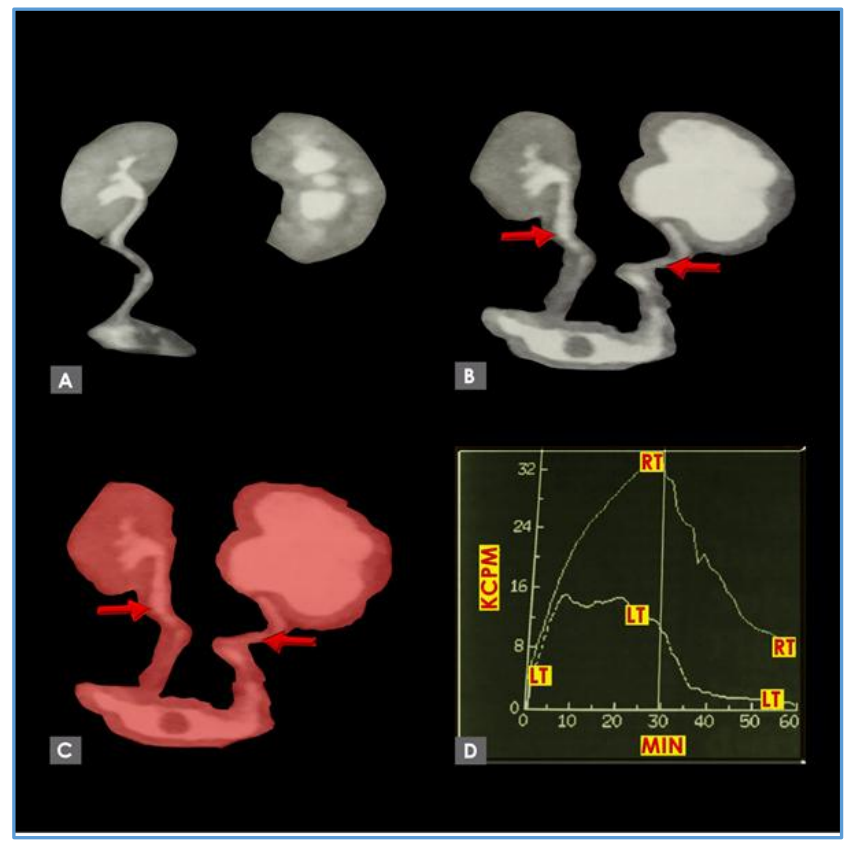

Fig 1(A-D):

(A). MR Urography contrast image showing 20 minutes, no left side ureter seen.

(B). MR Urography contrast 45 minutes MIP imag showing left UPJ obstruction with delayed ureter visualization.

(C). 3D MR Urography MIP Sequence showing coulur rendered left UPJ obstruction.

(D) Diurefic renogram showing left sided delayed renal function as curve is decreased.

Figure 1. Showing Left UPJ Obstruction as compared with MR Urography and Furosemide Nuclear Study.

Delay in Contrast RTT Time with Significant Elevation (RTT 450 seconds) is seen in both Studies.

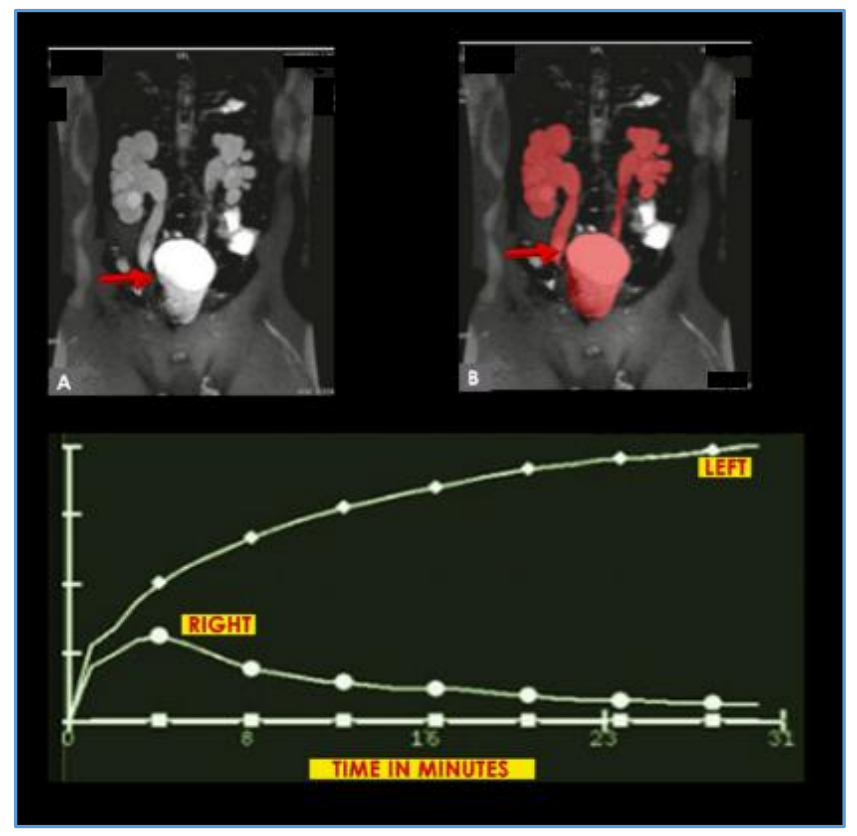

Fig 2(A-D):

(A). MR Urography contrast image showing 20 minutes, right distal ureter and VUJ not seen.

(B). MR Urography contrast 45 minutes colour MIP image conforming right VUJ obstruction and non visualized distal ureter.

(C). Diuretic renogram after 45 minutes conforming right renal curve dip due to distal level obstruction.

\section{Figure 2. Showing Right VUJ Obstruction as consistent} with Diuretic Renal Curve Analysis showing following

Peak Rise, Fall in Graph at Distal Level and Delay in Contrast Wash-Out

MRU provides an unprecedented level of anatomic information combined with quantitative functional evaluation of kidneys and urinary tracts in the evaluation of urinary stone disease in pregnancy. Because ionising radiation and contrast agents are not used, it is an attractive alternative to CT for people with contrast allergies, renal insufficiency, diabetes, pregnancy and young age. One consistent indication for MRU in flank pain is during pregnancy in case of recurrent flank pain. Compression, diuresis, enhanced agents and improvements in coil design, imaging time and combinations with other imaging techniques have provided us with higher resolution images and higher sensitivity for MRU in detecting urinary stones. Together with the presence of clinical data, it should be possible to make the correct MRU diagnosis of urolithiasis in the majority of patients.[9,5]

The major drawback of MRU is its low sensitivity in detecting non-obstructive or small obstructing calculi and parenchymal stones so there is still significant false negative rate, even though people has proposed some new techniques. ${ }^{[7,8,4]}$ MRU's other drawbacks are its relatively low accessibility and higher cost. We have not been able to define the best technique for MRU in diagnosing urinary tract calculi yet. However, MRU can be offered as an alternative to conventional urography and CT urography to avoid repetitive radiation exposure in patients with pregnancy or chronic urolithiasis.

\section{CONCLUSION}

MR Urography proved to be future gold standard for suspected mild pelvicaliectasis in our study. However, large 


\section{Jemds.com}

cohort study needs to be addressed to prove efficacy in large sample volume.

\section{ACKNOWLEDGEMENT}

We thank Venkatesan Zigma, PhD, for helping with statistical analysis.

\section{REFERENCES}

[1] Verswijvel GA, Oyen RH, Van Poppel HP, et al. Magnetic resonance imaging in the assessment of urologic disease: an all-in-one approach. Eur Radiol 2000;10(10):1614-9.

[2] Garcia-Valtuille R, Garcia-Valtuille AI, Abascal F, et al. Magnetic resonance urography: a pictorial overview. Br J Radiol 2006;79(943):614-26.

[3] Sigmund G, Stoever B, Zimmerhackl LB, et al. RAREMR-urography in the diagnosis of upper urinary tract abnormalities in children. Pediatr Radiol 1991;21(6):416-20.

[4] Rothpearl A, Frager D, Subramanian A, et al. MR urography: technique and application. Radiology 1995;194(1):125-30.

\section{Original Research Article}

[5] Nolte-Ernsting CCA, Adam GB, Gunther RW. MR urography: examination techniques and clinical applications. Eur Radiol 2001;11:355-72.

[6] Roy C, Saussine C, Jahn C, et al. Evaluation of RARE-MR urography in the assessment of ureterohydronephrosis. J Comput Assist Tomogr 1994;18(4):601-8.

[7] Aerts P, Van Hoe L, Bosmans H, et al. Breath hold MR urography using the HASTE technique. AJR Am J Roentgenol 1996;166(3):543-5.

[8] Regan F, Bohlman ME, Khazan R, et al. MR urography using HASTE imaging in the assessment of ureteric obstruction. AJR Am J Roentgenol 1996;167(5):111520.

[9] Ergen FB, Hussain HK, Carlos RC, et al. 3D excretory MR urography: improved image quality with intravenous saline and diuretic administration. J Magn Reson Imaging 2007;25(4):783-9. 